\title{
Cord blood-derived mesenchymal stem cells with hepatogenic differentiation potential ameliorate chronic liver affection in experimental models
}

\author{
Manal M. Kame ${ }^{1, A, B, D, E}$, Hanan G. El Baz ${ }^{1, A-C}$, Zeinab Demerdash ${ }^{1, A-C, F}$, Salwa Hassan ${ }^{1, B}$, Faten Salah ${ }^{1, B}$, Wafaa A. Mansour ${ }^{1, B, C}$, \\ Olfat Hammam ${ }^{2, B, C}$, Shimaa Atta ${ }^{1, B, C}$, Ali Bayoumi ${ }^{1, B,}$, , Marwa Hassan ${ }^{1, C-E}$, Soheir Mahmoud ${ }^{3, B}$ \\ 1 Department of Immunology, Theodor Bilharz Research Institute, Giza, Egypt \\ 2 Department of Pathology, Theodor Bilharz Research Institute, Giza, Egypt \\ ${ }^{3}$ Department of Parasitology, Theodor Bilharz Research Institute, Giza, Egypt \\ A - research concept and design; $B$ - collection and/or assembly of data; $C$ - data analysis and interpretation; \\ $\mathrm{D}$ - writing the article; $\mathrm{E}$ - critical revision of the article; $\mathrm{F}$ - final approval of the article
}

\section{Address for correspondence}

Manal M. Kamel

E-mail:baher_ronny@hotmail.com

\section{Funding sources}

Science and Technology Development Funds

(STDF), Cairo, Egypt

Conflict of interest

None declared

Received on January 6, 2017

Revised on March 23, 2017

Accepted on April 12, 2017
DOI

10.17219/acem/70430

\section{Copyright}

Copyright by Author(s)

This is an article distributed under the terms of the

Creative Commons Attribution Non-Commercial License

(http://creativecommons.org/licenses/by-nc-nd/4.0/)

\begin{abstract}
Background. The liver is one of the major target organs for which cell-based therapies are very promising. The limitations of various cellular therapies, including bone marrow (BM)-derived mesenchymal stem cells (MSCS), urges the exploration of stem cell sources more suitable for transplantation. Human umbilical cord blood (HUCB) can overcome these drawbacks with a favorable reparative outcome.
\end{abstract}

Objectives. The aim of this study was to evaluate the therapeutic potential of MSCs in 2 groups of chronic liver injury experimental models.

Material and methods. Propagation and characterization of MSCs isolated from cord blood (CB) samples were performed and differentiation into osteogenic, adipogenic and hepatogenic lineages was induced. The $1^{15 t}$ experimental model group ( 80 mice) included a negative control, a pathological control and 60 mice infected with Schistosoma mansoni (S. mansoni) and transplanted with MSCs. The $2^{\text {nd }}$ experimental model group (30 hamsters) included 10 healthy hamsters serving as a negative control and 20 hamsters injected with repeated doses of carbon tetrachloride $\left(\mathrm{CC}_{4}\right)$ to induce liver fibrosis; 10 of them were treated with an intrahepatic (IH) injection of $3 \times 10^{6} \mathrm{MSC}$ and the other 10 were untreated pathological controls. Mice and hamsters were sacrificed 12 weeks post-transplantation and their liver sections were stained immunohistochemically for the detection of human hepatocyte-like cells. Moreover, the sections were examined for the levels of fibrosis.

Results. In both models, the transplantation of CB-derived MSCS (CB-MSCS) resulted in the engraftment of the fibrotic livers with newly formed hepatocytes, as evidenced by positive immunohistochemistry staining with human Hepatocyte Paraffin 1 (Hep Par 1), alpha-fenoprotein (AFP), cytokeratin 18 (CK18), cytokeratin 7 (CK7), and OV6 monoclonal antibody. The transplanted liver sections showed markedly reduced hepatic fibrosis with a significantly lower fibrotic index, as well as significantly improved liver functions compared to the pathological control $(p<0.001)$.

Conclusions. This data provides hope that human CB-MSCs can be utilized as multipotent stem cells with unlimited potentiality in regenerative medicine and supports the concept of cellular therapy for the cure of hepatic fibrosis.

Key words: cord blood, mesenchymal stem cells, carbon tetrachloride, liver fibrosis 


\section{Introduction}

Liver fibrosis is the wound-healing reaction of hepatogenic cells to chronic liver injury. ${ }^{1}$ Following repetitive injury, the liver undergoes a remarkable tissue remodeling and develops fibrosis. This fibrosis is characterized by an extensive accumulation of the extracellular matrix, with the disposition of scar tissue encapsulating the region of injury. Although liver transplantation is undoubtedly the most efficient therapy for cirrhosis, broad clinical application of this procedure is limited by the scarcity of donor organs. ${ }^{2}$ Thus, it is crucial to investigate the efficacy of various treatments for hepatic cirrhosis.

The liver is one of the major target organs for which cell-based therapies are very promising. The limitations of various cellular therapies, including bone marrow (BM)-derived mesenchymal stem cells (MSCs), urges the exploration of stem cell sources more suitable for transplantation. Human umbilical cord blood (HUCB) can overcome these drawbacks with a favorable reparative outcome. ${ }^{3,4}$ Human umbilical cord blood stem cells remain viable even after long periods of cryopreservation. ${ }^{5-7}$ These cells are an ideal option for clinical applications due to their ready availability from cord blood (CB), ease of expansion in an in vitro culture, simple isolation via plastic adherence, ability to evade rejection, and their multipotentiality for differentiation. ${ }^{8,9}$

Over the past 2 decades, HUCB has been well established as a valued source of hematopoietic stem and progenitor cells. Besides hematopoietic cells, $\mathrm{CB}$ also contains cells with various stromal properties. ${ }^{10,11}$ From the point of view of clinical practice, it is very important to select a model of liver fibrosis that is close to the human disease in order to evaluate the effect of stem cells on the fibrosis. In the present study, we employed 2 models of fibrosis, induced by Schistosoma mansoni (S. mansoni) infection in mice and by carbon tetrachloride $\left(\mathrm{CCl}_{4}\right)$ in hamsters, to evaluate the effect of the MSCs injected through diverse routes and doses.

\section{Material and methods}

\section{Isolation and culture of mesenchymal stem cells}

Cord blood samples were collected from full-term babies delivered by cesarean section after informed written consent was given by the mothers, as approved by the institutional review board at Theodor Bilharz Research Institute (TBRI), Cairo, Egypt. Samples were processed according to the procedure described by Broxmeyer et al. ${ }^{10}$ Mononuclear cell fraction was isolated by the density gradient separation method and cultured according to the procedure described by Kögler et al. ${ }^{11}$ The MSCs were initiated in cultures, passaged and propagated according to Kögler et al. ${ }^{5}$

\section{Immunophenotyping}

Cells from the $3^{\text {rd }}$ passage were washed twice in phosphate-buffered saline buffer (PBS). Fluorescein isothiocyanate (FITC) mouse anti-human CD44, CD45 and HLA-DR, as well as FITC mouse anti-human CD105 coupled with PE (phycoerythrin) mouse anti-human CD90, and FITC mouse anti-human CD34 coupled with PE mouse antihuman CD73, were added to flow cytometry tubes.

One hundred microliters of the cell suspension was added to each tube and incubation was done for $20 \mathrm{~min}$ at $4^{\circ} \mathrm{C}$. The cells were washed twice with PBS and then resuspended in PBS. The final analysis was done by flow cytometer.

\section{Gene expression analysis}

Molecular analysis of the MSCs was performed according to the protocols described by Zaibak et al. and Demerdash et al. ${ }^{12,13}$ Total RNA was extracted from trypsinized cells using an RNeasy Mini Kit (Qiagen, Hilden, Germany). Its concentration and purity were assessed by a NanoDrop 2000 spectrophotometer (Thermo Fisher Scientific, Waltham, USA). Reverse transcripts were prepared using a high capacity cDNA kit (Applied Biosystems, Foster City, USA). The expression of the following genes was detected by real-time PCR using a QuantiTect SYBR Green PCR kit (Qiagen): Oct4 (5' TCTCGCCCCCTCCAGGT; 3'GCCCCACTCCAACCTGG), Sox2 (5' AGCTACAGCATGATGCAGGACC; 3'CTGGTCATGGAGTTGTACTGCAGG); and GAPDH (5'ATGGAGAAGGCTGGGGCTC, 3’AAGTTGTCATGGATGACCTTG).

\section{Osteogenic differentiation}

Following the guidelines of Kögler et al., the MSCs were seeded at a density of $6 \times 10^{3}$ cells $/ \mathrm{cm}^{2}$ into 6 -well plates and cultured in complete Dulbecco's modified Eagle's medium (DMEM). ${ }^{5}$ When the cells reached approx. 80-90\% confluency, the medium was replaced with osteogenic differentiation medium (Lonza, Basel, Switzerland). Dulbecco's modified Eagle's medium supplemented with $10 \%$ FBS, $1 \%$ penicillin/streptomycin and $1 \%$ L-glutamine was added to negative control wells. The media were replaced every 3-4 days. On the $14^{\text {th }}$ day, calcium deposition was assessed by alizarin red staining.

\section{Adipogenic differentiation}

Following the guidelines of Kögler et al., the MSCs were seeded at a density of $5 \times 10^{3}$ cells $/ \mathrm{cm}^{2}$ into 6 -well plates and cultured in complete DMEM. ${ }^{5}$ When the cells reached approx. 80-90\% confluency, they were cultured in the adipogenic induction medium for 3 days, followed by an adipogenic maintenance medium for 3 days. Three cycles of induction/maintenance were carried out for optimal adipogenic differentiation. Dulbecco's modified Eagle's 
medium supplemented with 10\% FBS 1\% penicillin/streptomycin, and 1\% L-glutamine was added to negative control wells. On the $21^{\text {st }}$ day, adipogenic differentiation was assessed by Oil Red O staining.

\section{Hepatogenic differentiation}

The induction of hepatogenic differentiation was done according to the protocol described by Waclawczyk et al. ${ }^{14}$ Briefly, the MSCs of passage 3 were seeded at a density of $5 \times 10^{3}$ cells $/ \mathrm{cm}^{2}$ and cultured in 30\% FBS/DMEM until reaching $80 \%$ confluency. The medium was then replaced with hepatogenic differentiation medium I (HDM I), consisting of hepatozyme supplemented with $0.5 \%$ FBS, hepatocyte growth factor (HGF) (50 ng/mL) (R\&D Systems, Minneapolis, USA), fibroblast growth factor-4 (FGF-4) (20 ng/mL) (R\&D Systems, Minneapolis, USA), epidermal growth factor (EGF) (20 ng/mL) (R\&D Systems, Minneapolis, USA), $1 \%$ penicillin/streptomycin, and $1 \% \mathrm{~L}$-glutamine. The cells were cultured for 2 weeks before exchanging the medium with HDM II, consisting of DMEM supplemented with HGF (50 ng/mL), FGF-4 (20 ng/mL), oncostatin-M (50 ng/mL), insulin transferrin selenium (ITS) $(5 \mu \mathrm{L} / \mathrm{mL}), 10^{-7}$ dexamethasone, $1 \%$ penicillin/ streptomycin, and $1 \%$ L-glutamine. The HDM II was left for another 2 weeks. Cells were scraped, collected and slide-fixed for cytopathological diagnosis of hepatocyte markers (glycogen, alpha-fetoprotein (AFP) and cytokeratin 18 (CK18)) using periodic acid-schiff (PAS) staining (Sigma-Aldrich, St. Louis, USA) for glycogen detection and immunohistochemistry staining for AFP and CK18 detection.

\section{Experimental model}

All animals were raised and maintained at the animal house in TBRI in barrier units with a defined and regularly monitored health status. All applicable international, national and/or institutional guidelines for the care and use of animals were followed.

\section{Induction of an experimental model for hepatic fibrosis in mice by infection with Schistosoma mansoni cercariae}

The S. mansoni-infected model consisted of $80 \mathrm{BALB} / \mathrm{c}$ mice, 8 weeks old and weighing 20-25 g, which were divided into the following groups:

- normal control group - 10 mice,

- infected control group - 10 mice infected with S. mansoni cercariae (60 cercariae/mouse) using the tail immersion method and left for 12 weeks to induce liver fibrosis, and

- MSCs-transplanted group - 60 mice infected with S. mansoni. This group was subdivided according to the method of MSCs transplantation into 2 subgroups (30 mice each): intravenous (IV) and intrahepatic (IH).
Both the IV and IH subgroups were further subdivided, according to the dose of transplanted MSCs, into 3 subgroups; IV1, IH1, IV2, IH2, IV3, and IH3, transplanted with $3 \times 10^{5} \& 6 \times 10^{5} \& 1 \times 10^{6} \mathrm{MSCs} /$ mouse, respectively.

Mice from each group were sacrificed 4,8 , and 12 weeks after MSCs transplantation, respectively.

\section{Induction of experimental model for hepatic cirrhosis} by carbon tetrachloride

The $\mathrm{CCl}_{4}$ model consisted of 30 hamsters weighing 60-65 g and divided into 3 groups:

- normal control group - 10 healthy hamsters;

- $\mathrm{CCl}_{4}$ control group - 10 hamsters injected with $\mathrm{CCl}_{4}$ at a dose of $100 \mu \mathrm{L} /$ hamster (Al-gamhuria, Cairo, Egypt) mixed with olive oil $\left(100 \mu \mathrm{LCCl}_{4}+900 \mu \mathrm{L}\right.$ olive oil) intraperitoneally (IP) twice a week for 2 months. ${ }^{15}$ Healthy control hamsters were injected with the same volume of olive oil;

- MSCs-transplanted group - 10 hamsters injected with $\mathrm{CCl}_{4}$ as above and treated with MSCs $\left(3 \times 10^{6}\right.$ cells/ hamster) IH 2 months after $\mathrm{CCl}_{4}$ injection.

The cells were injected via the IH route because hamsters have no tail and the IV route was not feasible. The hamsters were sacrificed 12 weeks after MSCs transplantation.

\section{Liver function analysis}

Serum aspartate aminotransferase (AST), alanine aminotransferase (ALT), albumin (ALB), and bilirubin were measured using a Synchron CX5 (Beckman Coulter, Brea, USA) analyzer.

\section{Liver pathology and immunohistochemistry analysis}

Liver sections from sacrificed animals were stained with hematoxylin and eosin (H\&E) for histologic assessment and with Sirius red for assessment of fibrosis. ${ }^{16}$ The CK18, cytokeratin 7 (CK7), Hepatocyte Paraffin 1 (Hep Par 1), AFP, and OV6 monoclonal antibodies were used (Santa Cruz Biotechnology Company, Santa Cruz, USA). In addition, negative controls in which the primary antibody was omitted and replaced by PBS were also used. Livers known to express markers were used as positive controls.

The intensity, distribution and pattern were analyzed and immunoexpression was evaluated. The percentage of positively stained cells was determined semiquantitatively by assessing the whole section.

\section{Liver fibrosis analysis}

Hepatic sections, $20 \mu \mathrm{m}$ in thickness, were prepared from paraffin sections and stained with Picrosirius red. For the quantification of the collagen content, an Automatic Computer Image Analysis System (Zeiss, Oberkochen, Germany) was used. Image analysis was performed using 
the computer software AxioVision v. 4.8, supplied by the manufacturer of the system. The sectional area of the red-stained fibrous tissue was measured in 5 consecutive microscopic fields ( $\times 5$ magnification) to yield the fibrotic area $\left(\mu \mathrm{m}^{2}\right)$ and the proportion of fibrotic area relative to the total area examined was then calculated (fibrotic index in \%).

\section{Statistical analysis}

The data were analyzed using the SPSS package v. 18.0 for Windows (SPSS Inc., Chicago, USA). Laboratory data of different groups were compared with one-way analysis of variance (ANOVA). At a p-value $\leq 0.05$ differences were considered statistically significant.

\section{Results}

\section{Immunophenotyping}

The MSCs showed high expression levels of adhesion marker CD44, typical mesenchymal markers (CD90 and CD73), the endoglin receptor CD105, and dual expression of CD105/90; they were negative for HLA-DR, hematopoietic lineage marker CD34 and leukocyte common antigen CD45 expression (Table 1).

\section{Gene expression analysis}

The MSCs expressed Oct4 and Sox2, which are considered to be core transcription factors that regulate the maintenance of the pluripotent state in embryonic and adult stem cells.

\section{Osteogenic differentiation}

When HUCB-MSCs were induced to differentiate into osteogenic lineage, the spindle shape of the HUCB-derived cells became less elongated and more polygonal in shape with the formation of aggregates (Fig. 1A).

\section{Adipogenic differentiation}

Under the influence of adipogenic differentiation conditions, the MSCs became large and rounded with an accumulation of neutral lipid vacuoles indicated by the Oil Red O stain (Fig. 1B).

\section{Hepatogenic differentiation}

\section{Morphology}

The fibroblastic-like morphology of the MSCs was lost. A broadened, flattened shape developed at first, while a round shape with a cuboidal morphology developed later. Slides coated with MSCs-derived cells and stained with $\mathrm{H} \& \mathrm{E}$ are shown in Fig. $1 \mathrm{C}$.

\section{Periodic acid-schiff staining}

Smears were examined using a Zeiss Axio Scope A1 (Zeiss), under a magnification of $\times 400$. Glycogen storage was determined by PAS staining. Positively stained glycogen granules were detected in the cytoplasm of differentiated MSCs, while undifferentiated MSCs were negative for PAS staining (Fig. 2).

\section{Immunocytochemistry}

Smears were examined using a Zeiss Axio Scope A1 (Zeiss), under $\times 400$ magnification. With hepatocyte markers (AFP and CK18) being cytoplasmic markers, both AFP and CK18 were expressed in the cytoplasm of differentiated MSCs (Fig. 3 A,B), while undifferentiated MSCs were negative for both markers.

\section{Schistosoma mansoni-induced fibrogenesis model}

Only the results of transplantation with $1 \times 10^{6} \mathrm{MSCs}$ after 3 months are presented here, as they were the most promising results.

Effect of intravenous transplantation of $1 \times 10^{6} \mathrm{MSCs} /$ mouse on Schistosoma mansoni-induced fibrogenesis

Intravenous transplantation of $1 \times 10^{6} \mathrm{MSCs}$ in S. mansoni-infected mice revealed a high, significant reduction ( $\mathrm{p}<0.001)$ in fibrotic index 3 months after MSCs transplantation, compared to the corresponding index in the infected control group (Table 1).

Table 1. Results of liver functions - aspartate aminotransferase (AST), alanine aminotransferase (ALT) and albumin (ALB) levels - and \% fibrosis in different studied groups of murine Schistosoma mansoni-infected mice (mean $\pm \mathrm{SE}$ )

\begin{tabular}{|c|c|c|c|c|}
\hline \multirow[b]{2}{*}{ Parameter } & \multicolumn{4}{|c|}{ Studied groups of murine Schistosoma mansoni-infected mice } \\
\hline & negative control $(n=10)$ & infected control $(n=10)$ & $\begin{array}{c}\text { infected \& treated with IV } \\
1 \times 10^{6} \operatorname{MSCs}(n=30)\end{array}$ & $\begin{array}{c}\text { infected \& treated with IH } \\
1 \times 10^{6} \mathrm{MSCS}(\mathrm{n}=30)\end{array}$ \\
\hline Albumin $[\mathrm{g} / \mathrm{dL}]$ & $4.36 \pm 0.049$ & $0.73 \pm 0.18$ & $5.2 \pm 0.167$ & $3.2 \pm 0.096$ \\
\hline $\mathrm{ALT}[\mathrm{U} / \mathrm{L}]$ & $24.66 \pm 0.421$ & $136 \pm 3.65$ & $51 \pm 0.96$ & $66.82 \pm 3.06$ \\
\hline AST [U/L] & $68.67 \pm 0.76$ & $350.67 \pm 19.33$ & $79.67 \pm 7.02$ & $101.76 \pm 0.66$ \\
\hline$\%$ fibrosis & $0.2 \pm 0.04$ & $27.48 \pm 0.745$ & $5.37 \pm 0.21$ & $7.58 \pm 0.38$ \\
\hline
\end{tabular}



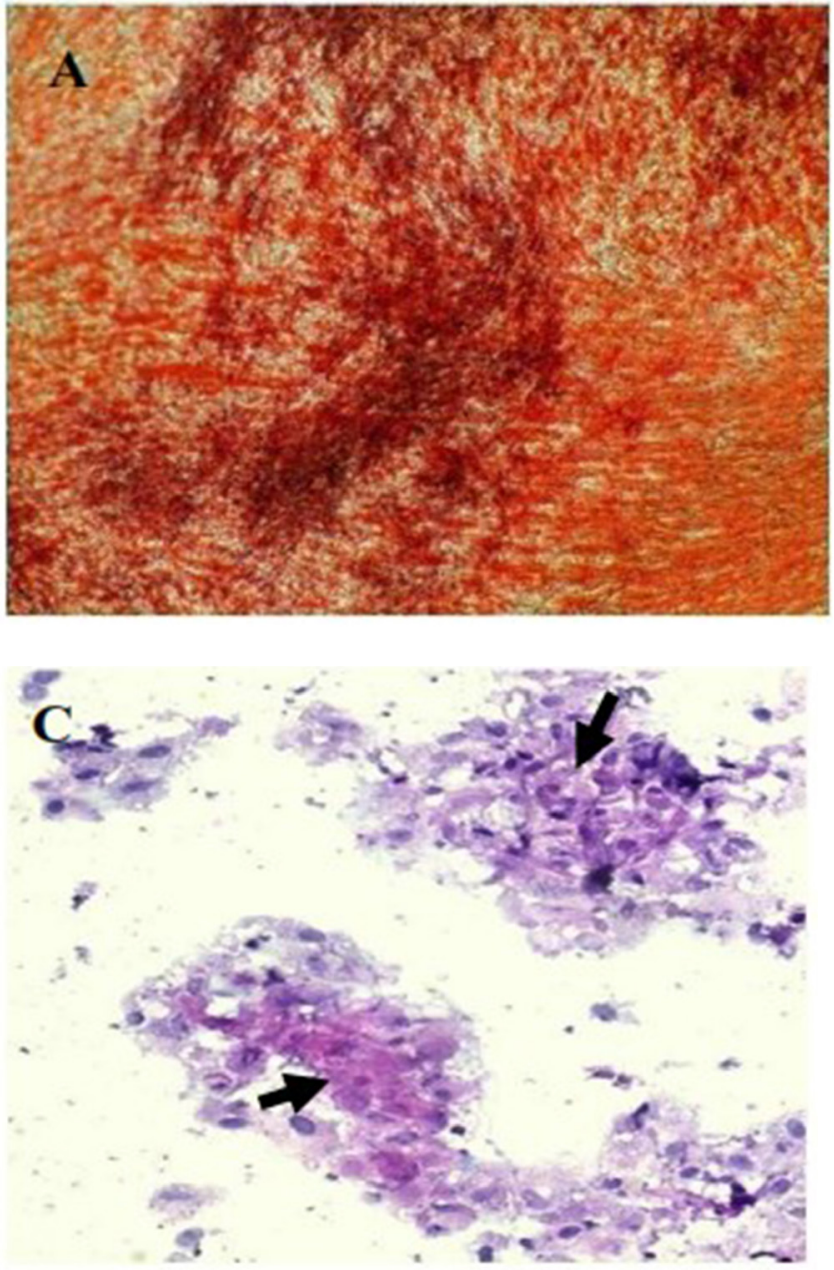

Fig. 1. Mesenchymal stem cells (MSCs) of P3 were induced to osteogenic adipogenic and hepatogenic differentiation, which was confirmed A) by the formation of a mineralized matrix as evidenced by alizarin red staining, B) by the accumulation of neutral lipid vacuoles evidenced by the oil red $\mathrm{O}$ stain, and $\mathrm{C}$ ) by the morphological changes towards hepatocyte-like cells showing large polyhedral cells with rounded nuclei in small sheets or separate hepatocyte-like cells

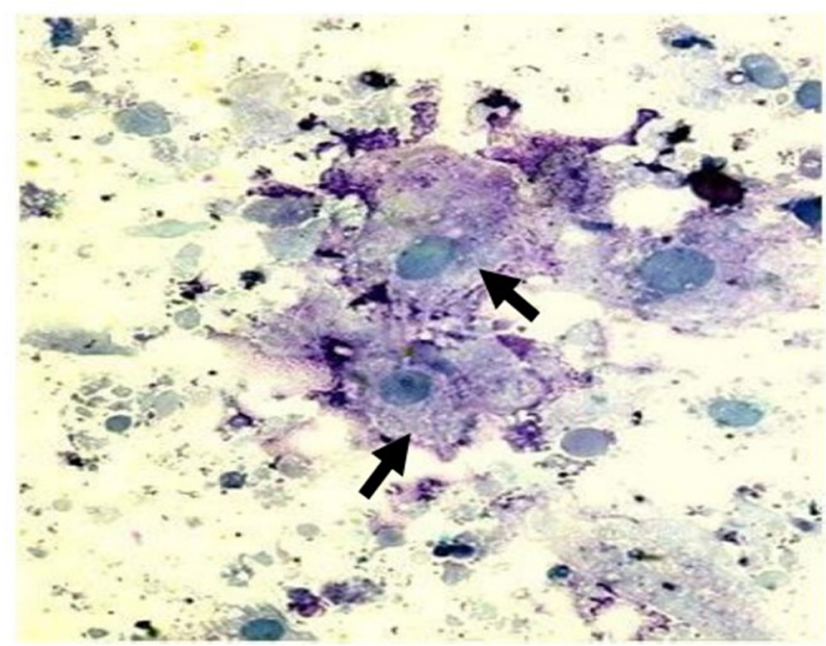

Fig. 2. Positively periodic acid-Schiff (PAS)-stained glycogen granules in the cytoplasm of differentiated mesenchymal stem cells (MSCs) showing large polyhedral cells with rounded nuclei in small sheets or separate hepatocytes-like cells positive for PAS stain ( $\times 400$ magnification)

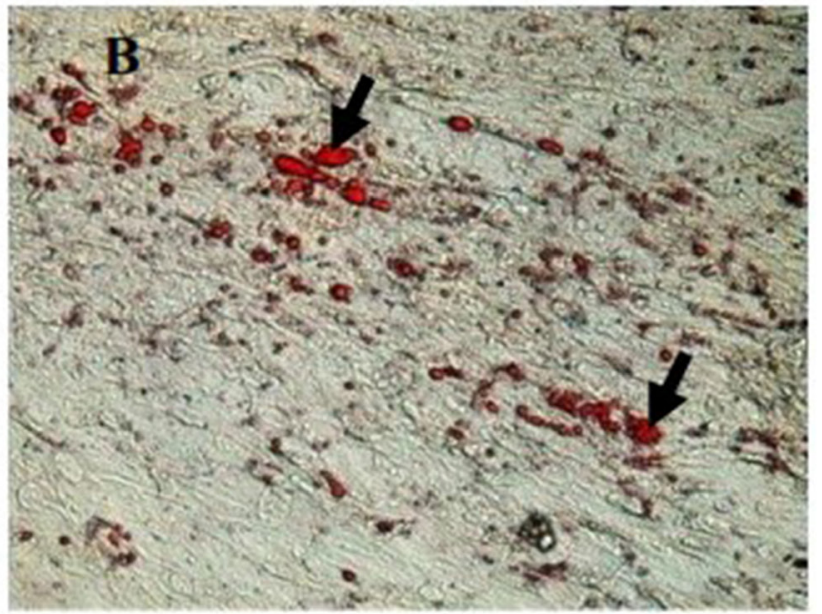

There was a significant improvement $(\mathrm{p}<0.001)$ in the ALT, AST and ALB (Table 1) levels of the MSCstransplanted group 3 months post-transplantation, when compared to the corresponding level in the infected control. It was observed that ALB reached a nearly normal level after the $3^{\text {rd }}$ month.

In mice transplanted IV with $1 \times 10^{6} \mathrm{MSCs}$ and sacrificed 12 weeks after transplantation, liver sections stained with H\&E (Fig. 4 A-C) and Sirius red (Fig. 4 D-F) showed diminished granuloma size and a relative decrease in hepatic fibrosis. The cells were able to engraft into the fibrotic livers with some signs of regeneration, i.e., newly formed hepatocytes of human origin.

Livers of the MSCs-transplanted group showed engraftment with human hepatocyte-like cells, as proven by the cytoplasmic expression of AFP, Hep Par 1, CK18, CK7, and OV6 (Fig. 5 A-E) and by the negative staining of hepatocytes for desmin and vimentin. In addition, the livers of the MSCs-transplanted group showed less fibrosis than the pathological control group.

\section{Effect of intrahepatic transplantation of $1 \times 10^{6} \mathrm{MSCs} /$ mouse on Schistosoma mansoni-induced fibrogenesis}

Almost same results were obtained as in IV route groups with less reduction in the fibrotic index (Table 1).

There was a significant improvement in ALT, AST and ALB levels (Table 1) 3 months after IH MSCs transplantation, compared to the levels in the infected control group. This improvement was less marked than that with the IV route groups.

In mice transplanted intrahepatically with $1 \times 10^{6} \mathrm{MSCs}$, liver sections stained with either $\mathrm{H} \& \mathrm{E}$ or Sirius red showed relatively diminished granuloma size and a relative decrease in hepatic fibrosis (Fig. 4), compared to the IV-transplanted group.

Immunoperoxidase staining of the liver sections from $\mathrm{BALB} / \mathrm{c}$ mice infected with $\mathrm{S}$. mansoni and injected $\mathrm{IH}$ 


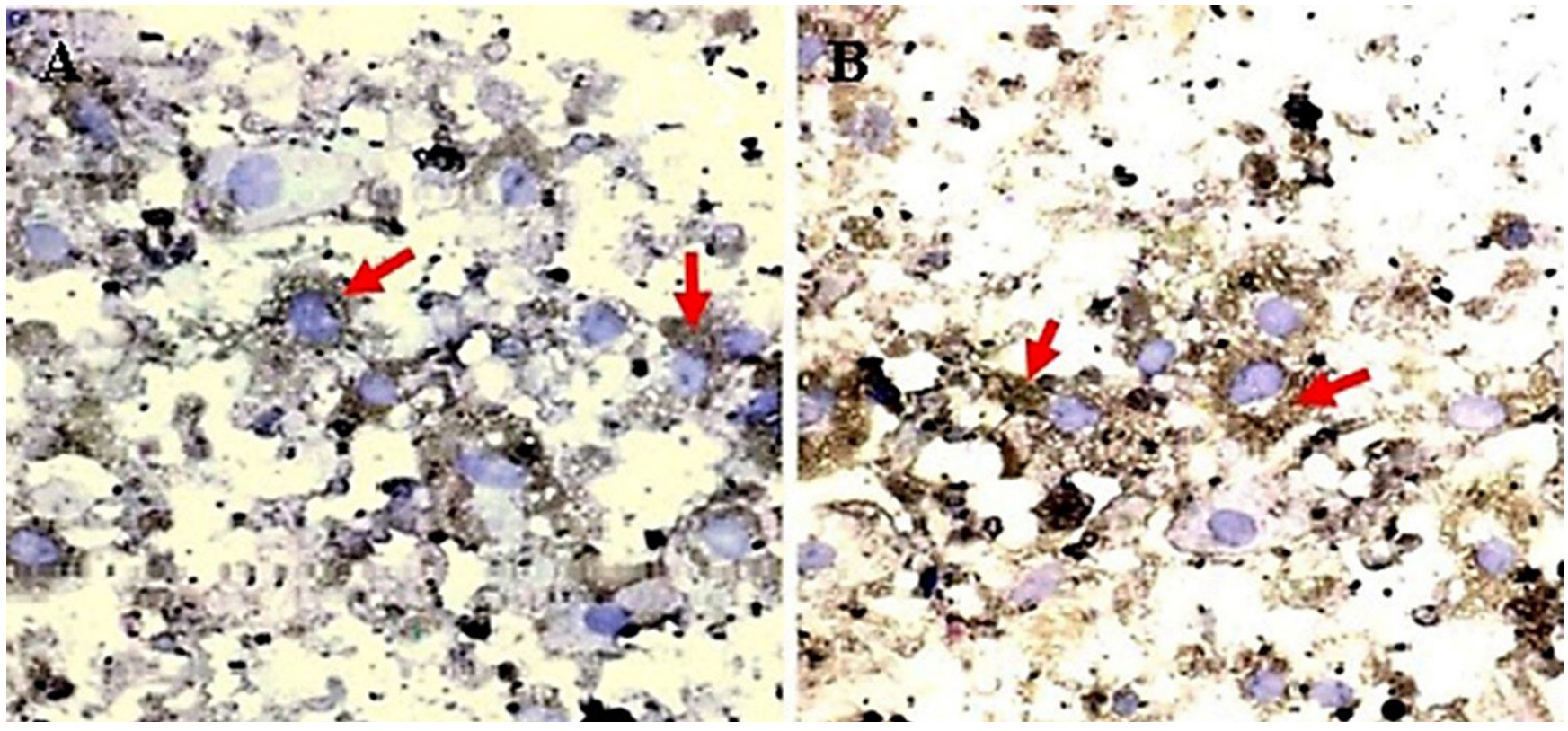

Fig. 3. Mesenchymal stem cells (MSCs)-differentiated hepatocyte-like cells positive for A) alpha-fetoprotein (AFP) and B) cytokeratin 18 (CK18), presented as cytoplasmic brownish stain (immunohistochemistry (IHC) $\times 400$ magnification)
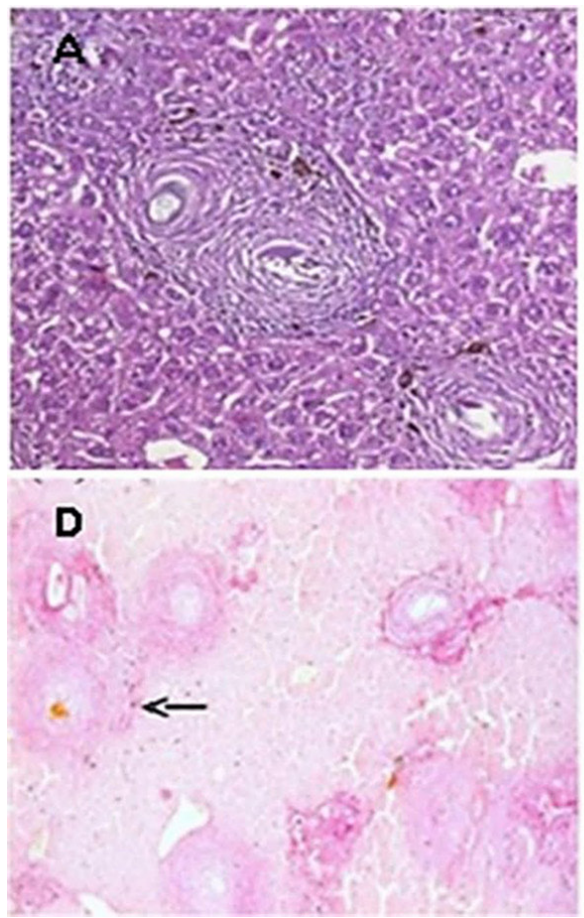
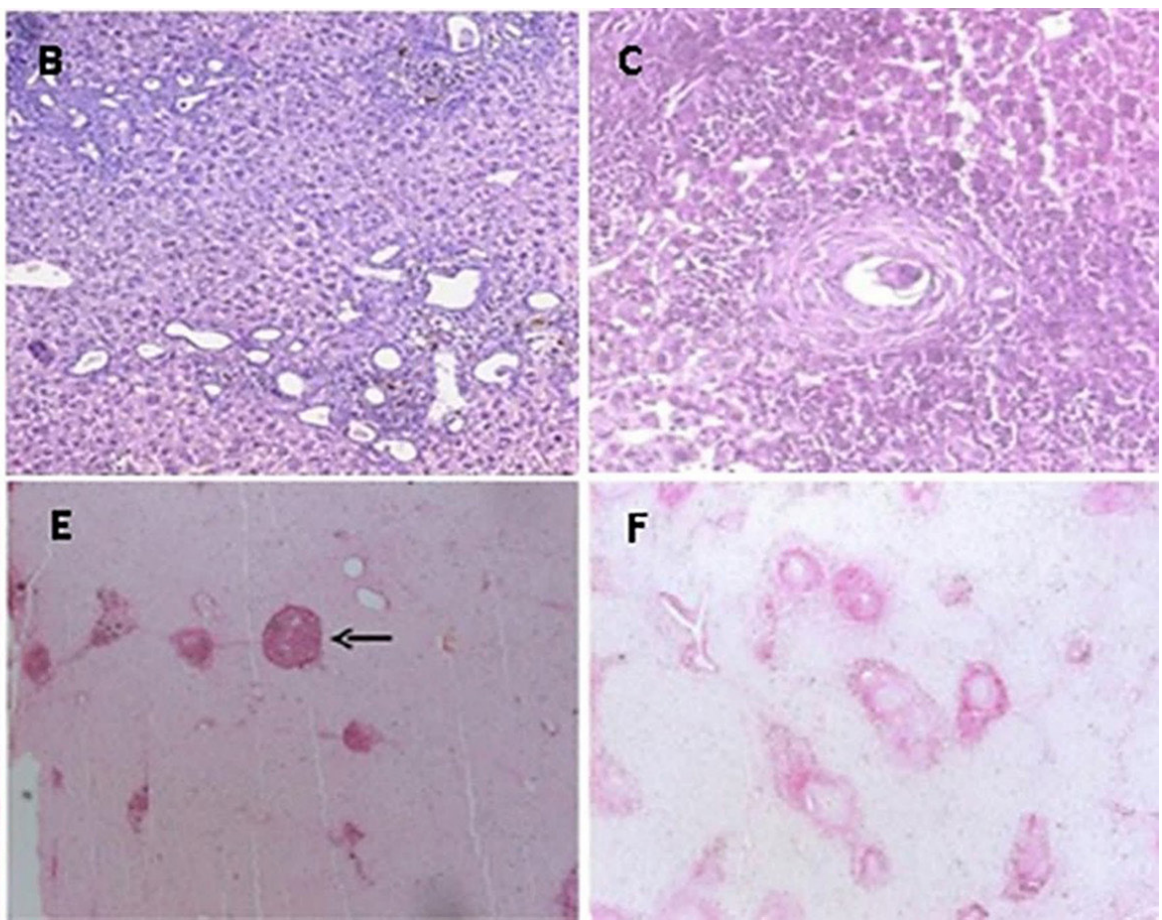

Fig. 4. Liver sections from BALB/c mice (hematoxylin and eosin (H\&E) staining, $\times 400$ magnification): A) infected control mouse showing a large fibrocellular granuloma 8 weeks post-infection; B) infected \& intravenously (IV) $1 \times 10^{6}$ mesenchymal stem cells (MSCs)-treated mice 12 weeks post-treatment, showing a small regressed fibrocellular granuloma; C) infected and and intrahepatically (IH) $1 \times 10^{6}$ MSCs-treated mice 12 weeks post-treatment, showing moderate collagen fibrous bundles forming moderate size fibrocellular bilharzial granuloma. Liver sections from BALB/c mice infected with Schistosoma mansoni (Sirius red staining), showing: D) a large untreated fibrocellular granuloma 8 weeks post-infection; E) IV $1 \times 10^{6}$ MSCs-treated mice 12 weeks post-treatment with small regressed granuloma; F) IH $1 \times 10^{6} \mathrm{MSC}$-treated mice 12 weeks post-treatment, showing moderate size fibrocellular bilharzial granuloma 

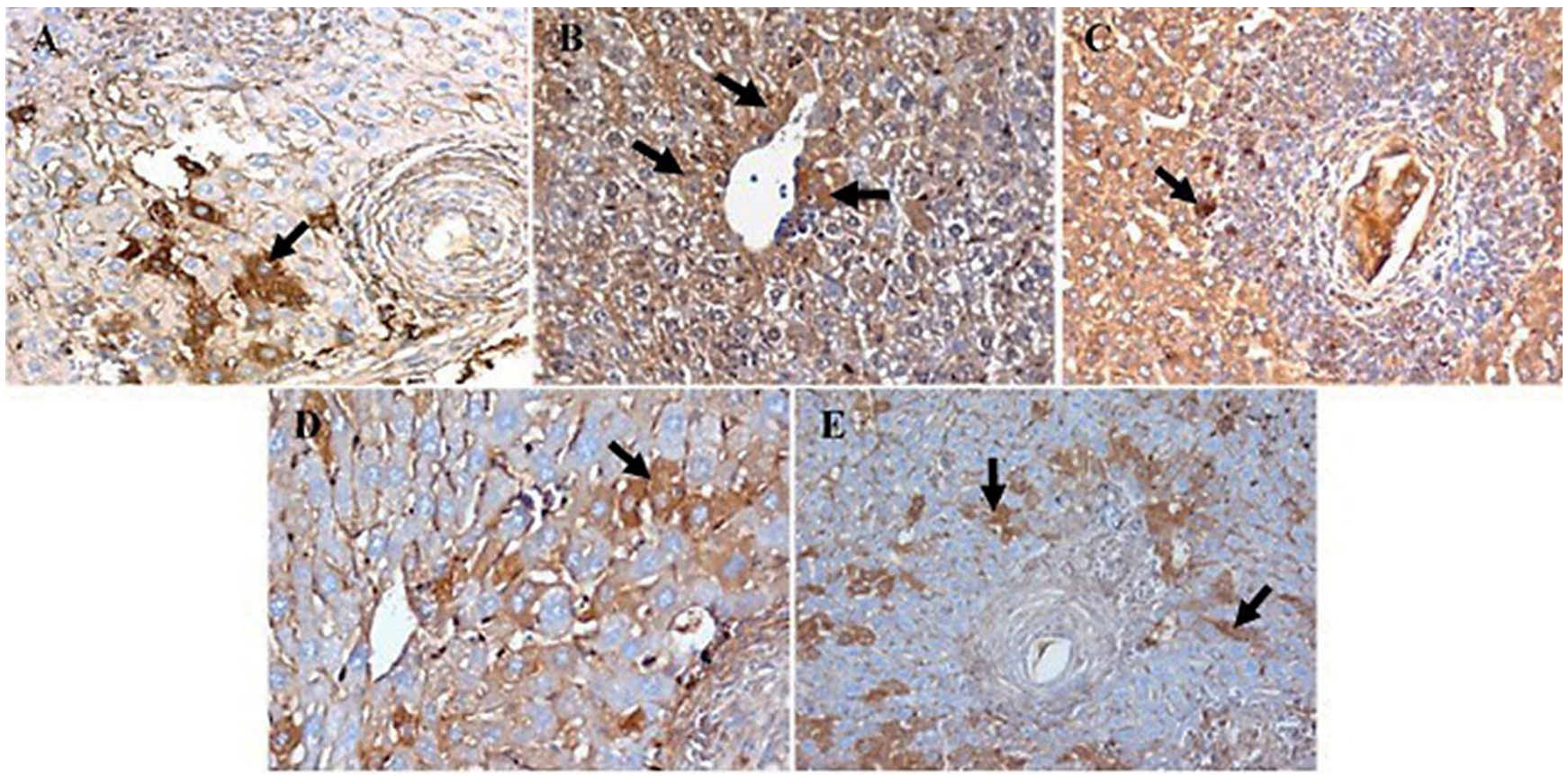

Fig. 5. Immunoperoxidase staining ( $\times 400$ magnification) of liver sections of Schistosoma mansoni-infected BALB/c mice 12 weeks post-intravenous (IV) treatment with $1 \times 10^{6}$ mesenchymal stem cells (MSCs), showing: A) 50\% new hepatocytes positive for alpha-fetoprotein (AFP), as a brownish cytoplasmic stain; B) 50\% positivity for hepatocyte paraffin 1 (Hep Par 1); C) 50\% positivity for cytokeratin 18 (CK18); D) 40\% positivity for cytokeratin 7 (CK7); and E) $45 \%$ positivity for OV6
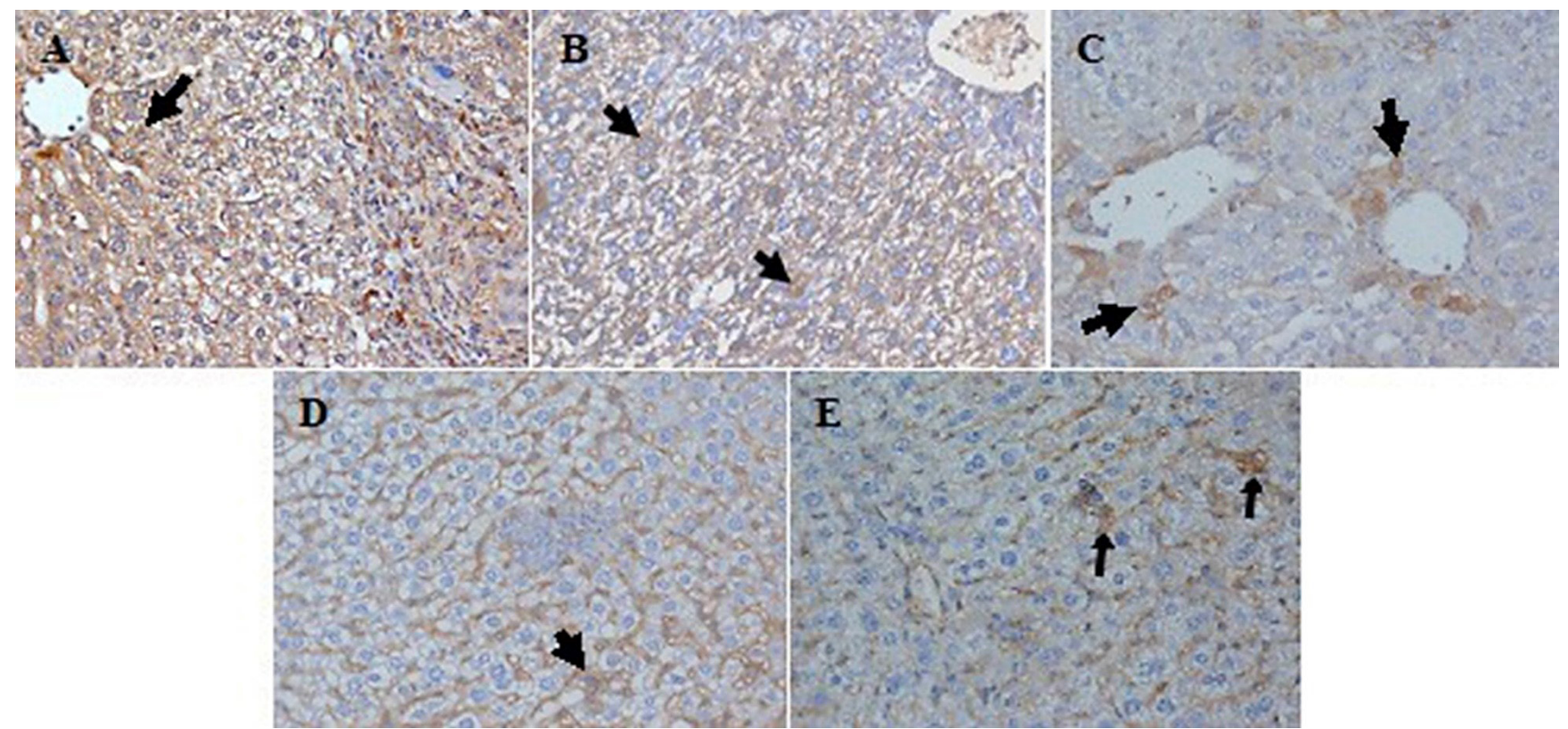

Fig. 6. Immunoperoxidase staining ( $\times 400$ magnification) of liver sections of Schistosoma mansoni-infected BALB/c mice 12 weeks post-intrahepatic (IH) treatment with $1 \times 10^{6}$ mesenchymal stem cells (MSCs) showing: A) 10\% new hepatocytes positive for alpha-fetoprotein (AFP), as a brownish cytoplasmic stain; B) 15\% positivity for hepatocyte paraffin 1 (Hep Par 1); C) 20\% positivity for cytokeratin 18 (CK18); D) 15\% positivity for cytokeratin 7 (CK7); and E) 10\% positivity for OV6 
with $1 \times 10^{6}$ MSCs 12 weeks post-treatment showed new hepatocytes with brownish cytoplasmic stain positive for AFP, Hep Par 1, CK18, CK7, and OV6 (Fig. 6 A-E). Cells were able to engraft into the fibrotic liver with evidence of regeneration in the newly formed hepatocytes of human origin.

\section{Carbon tetrachloride-induced liver cirrhosis model}

Six trials over 3 months were performed to optimize the protocol for developing a model of cirrhosis. Two models were subjected to different doses and concentrations of $\mathrm{CCl}_{4}$ to reach a sub-lethal dose which induced liver cirrhosis, yet avoided exposing the liver to severe toxicity ending in the death of the animal.

$\mathrm{BALB} / \mathrm{c}$ mice were used as the $1^{\text {st }}$ model. Carbon tetrachloride was dissolved in olive oil in ratios of 1:1, 1:2 and 1:5. The animals were then injected IP twice a week with $0.5 \mathrm{~mL} / \mathrm{kg}, 0.5 \mathrm{~mL} / \mathrm{kg}$ and $0.1 \mathrm{~mL} / \mathrm{kg}$ of the prepared concentrations, respectively. Severe liver affection and death occurred within 1, 2 and 3 weeks, respectively.

The $2^{\text {nd }}$ model used was hamsters. Carbon tetrachloride was dissolved in olive oil in ratios of 1:20, 1:5 and 1:10. The animals were injected IP twice a week with $0.01 \mathrm{~mL} / \mathrm{kg}$, $0.1 \mathrm{~mL} / \mathrm{kg}$ and $0.0016 \mathrm{~mL} / \mathrm{kg}$ of the prepared concentrations, respectively.

Liver specimens obtained from the animals injected with $0.01 \mathrm{~mL} / \mathrm{kg}$ of the emulsion at a ratio of 1:20 did not show any evidence of liver affection or fibrosis after 1 month, while the hamsters subjected to a dose of $0.1 \mathrm{~mL} / \mathrm{kg}$ at a ratio of 1:5 died as a result of severe liver affection within 2-3 weeks.

Histopathologic studies of liver specimens obtained from hamsters injected with $100 \mu \mathrm{L} /$ hamster of the emulsion at a ratio of 1:10 showed that fibrosis started to be evident within 1 month and complete cirrhotic nodules were found after 3 months.

\section{Carbon tetrachloride-induced fibrogenesis in hamsters transplanted intrahepatically with $3 \times 10^{6}$ mesenchymal stem cells}

Transplantation of $3 \times 10^{6} \mathrm{MSCs}$ in $\mathrm{CCl}_{4}$-injected hamsters revealed a significant reduction $(p<0.01)$ in the fibrotic index 3 months after MSCs transplantation, compared to the corresponding index in the $\mathrm{CCl}_{4}$ control group (Table 2).

There was a high significant improvement $(\mathrm{p}<0.001)$ in ALT, AST and bilirubin levels (Table 2) 3 months after MSCs transplantation, compared to the corresponding levels in the $\mathrm{CCl}_{4}$ control group.

The liver sections of the $\mathrm{CCl}_{4}$ control hamsters stained with either H\&E or Sirius red showed micro- and macrocirrhotic nodules (Fig. 7 A,B). Diminished cirrhotic nodules and a relative decrease in hepatic fibrosis were observed after MSCs transplantation. Cells were able to engraft into the fibrotic livers with newly formed hepatocytes of human origin as an evidence of some regeneration (Fig. 7 C,D).

The livers of the MSCs-transplanted group showed engraftment with human hepatocyte-like cells as proven by cytoplasmic expression of human AFP, Hep Par 1, CK18, OV6, and CK7 (Fig. 8 A-E).

\section{Discussion}

In the current study, CB-MSCs were isolated and expanded, and were positive for MSC phenotypic markers (CD73, CD105, CD90, and CD44), while being negative for HLA-DR and the hematopoietic stem cell phenotypic markers (CD34 and CD45). They showed high expression levels of Oct4 and Sox2 transcription factors, which regulate the maintenance of the pluripotency state in embryonic and adult stem cells. ${ }^{17,18}$ Moreover, MSCs showed multilineage differentiation potential, being able to differentiate in vitro into mesodermal lineage cells (osteoblasts and adipocytes), and into the hepatogenic lineage of an endodermal origin. The expression of hepatic parenchymal markers - ALB, AFP, CK18, and glycogen - showed that our MSCs cultures contained functional hepatocytes, similar to the outcomes of the studies by Liang et al. and Li et al. ${ }^{19,20}$

We assessed the therapeutic effect of the transplantation of MSCs in 2 different experimental models: mice with S. mansoni infection (either IV or IH) and $\mathrm{CCl}_{4}$-injected hamsters. In cell-based therapies, the dose of the transplanted cells is a key factor, as an appropriate cell count is vital for the survival of the injured experimental animals. In this study, we tested 3 doses $\left(3 \times 10^{5}, 6 \times 10^{5}\right.$ and $1 \times 10^{6}$ ) of MSCs/mouse and a dose of $3 \times 10^{6} \mathrm{MSCs} /$ hamster, in $0.5 \mathrm{~mL}$ DMEM. The recipient mice tolerated all doses and the best results were obtained when using the highest dose of $1 \times 10^{6} /$ mouse. It was previously reported that the therapeutic effects of MSCs on liver cirrhosis gradually improved with increased cell dose. ${ }^{21} \mathrm{~A}$ dose

Table 2. Results of liver functions (ALT, AST and total bilirubin) and $\%$ fibrosis in different studied groups of the $\mathrm{CCl}_{4}$ model (mean $\pm \mathrm{SE}$ )

\begin{tabular}{|l|c|c|c|}
\multirow{2}{*}{\multicolumn{1}{c|}{ Parameter }} & \multicolumn{3}{|c|}{ Studied groups of $\mathrm{CCl}_{4}$ model } \\
\cline { 2 - 4 } & $\begin{array}{c}\text { negative } \\
\text { control } \\
(\mathrm{n}=10)\end{array}$ & $\begin{array}{c}\mathrm{CCl}_{4} \text { group } \\
(\mathrm{n}=10)\end{array}$ & $\begin{array}{c}\mathrm{CCl}_{4} \& \text { treated } \\
\text { with IH } \\
3 \times 10^{6} \mathrm{MSCs} \\
(\mathrm{n}=10)\end{array}$ \\
\hline Total bilirubin $[\mathrm{mg} / \mathrm{dL}]$ & $0.28 \pm 0.02$ & $5.97 \pm 0.23$ & $0.52 \pm 0.01$ \\
\hline ALT [U/L] & $31.3 \pm 2.03$ & $687.67 \pm 13.3$ & $127.67 \pm 1.76$ \\
\hline AST [U/L] & $74.3 \pm 2.3$ & $901.7 \pm 6.6$ & $102.3 \pm 1.45$ \\
\hline \% fibrosis & $1.27 \pm 0.19$ & $17 \pm 0.82$ & $2 \pm 0.21$ \\
\hline
\end{tabular}

AST - aspartate aminotransferase; ALT - alanine aminotransferase; $\mathrm{CCl}_{4}$ - carbon tetrachloride; $\mathrm{IH}$ - intrahepatic; MSCs - mesenchymal stem cells; SE - standard error. 

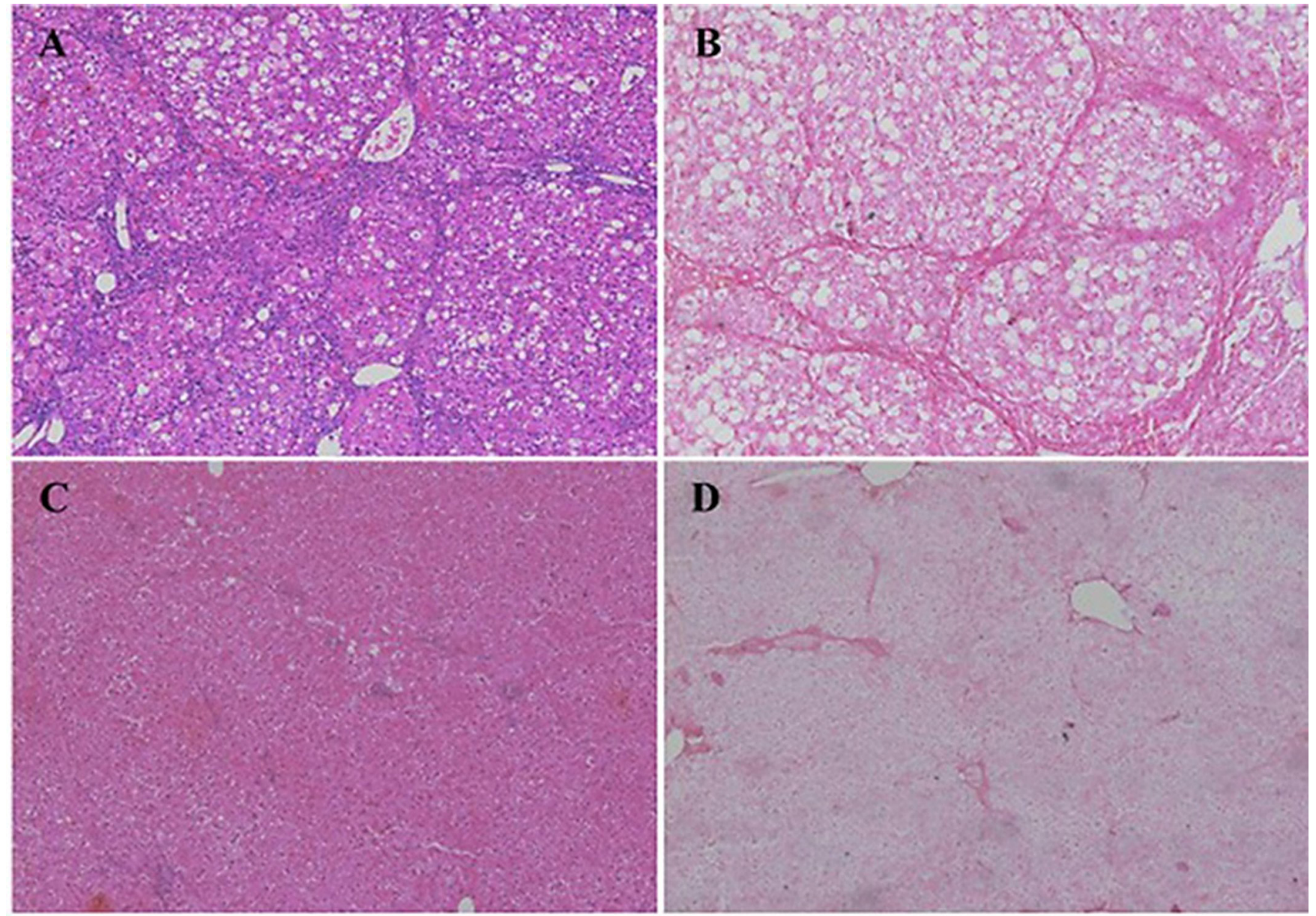

Fig. 7. Liver sections of a carbon tetrachloride (CCl $)_{4}$ control hamster showing micro- and macrocirrhotic nodules: A) hematoxylin and eosin (H\&E) staining, $\times 200$ magnification; B) Sirius red, $\times 200$ magnification. Liver sections of $\mathrm{CCl}_{4}$ hamster 12 weeks post-intrahepatic (IH) treatment with $3 \times 10^{6}$ mesenchymal stem cells (MSCs), showing few fibrous bundles, liver with almost intact architecture; C) H\&E, x100 magnification; and D) Sirius red, ×200 magnification

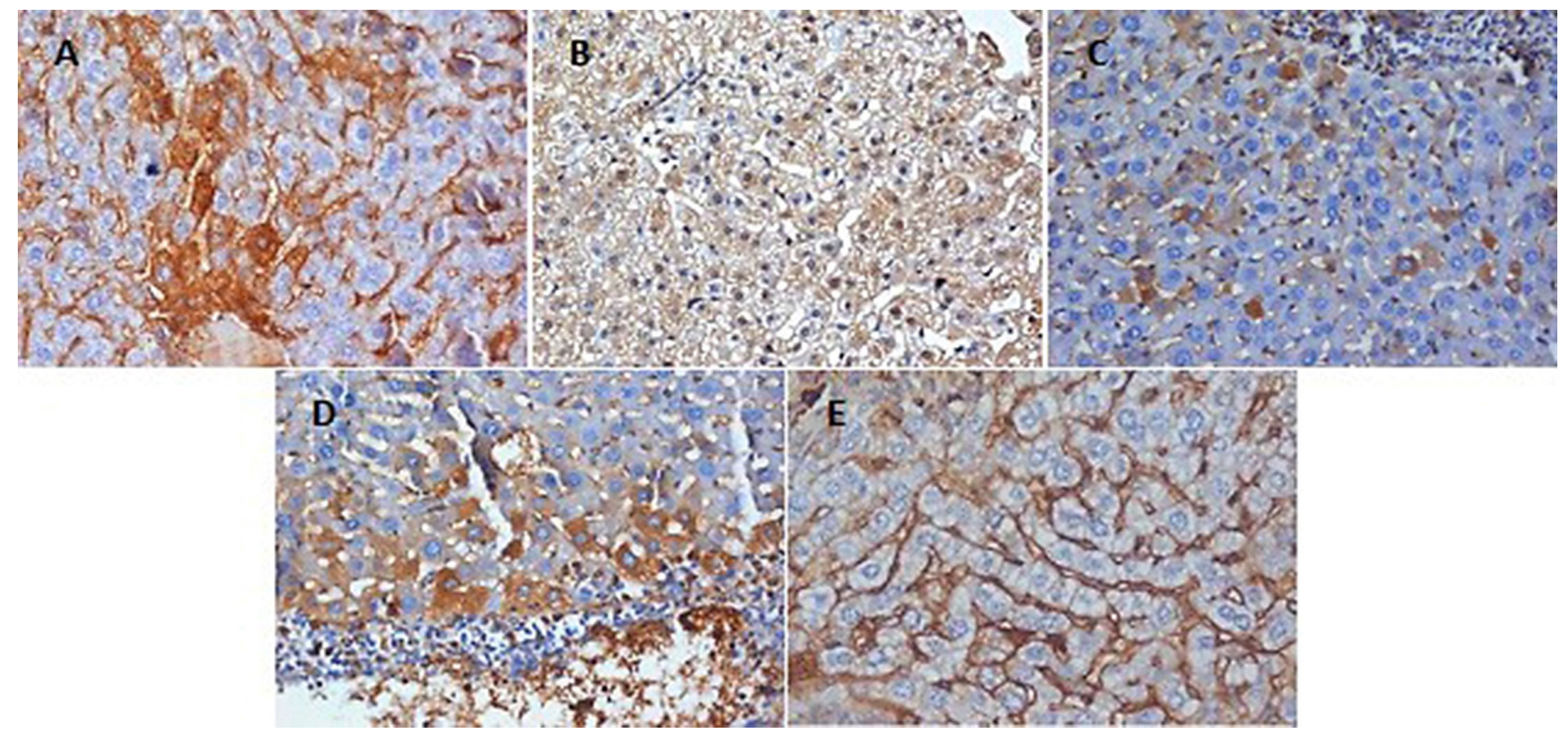

Fig. 8. Immunoperoxidase staining ( $\times 400$ magnification) of liver sections of carbon tetrachloride $\left(\mathrm{CCl}_{4}\right)$ hamster 12 weeks post-intrahepatic $(\mathrm{IH})$ mesenchymal stem cells (MSCs)-treatment, showing positive brownish cytoplasmic staining for A) alpha-fetoprotein (AFP), B) hepatocyte paraffin 1 (Hep Par 1), C) cytokeratin 18 (CK18), D) OV6, and E) cytokeratin 7 (CK7) 
of $1 \times 10^{6} \mathrm{MSCs} / \mathrm{mouse}$ has been used in several mice experiments. ${ }^{22,23}$ However, Yang et al. found that $1 \times 10^{6}$ cells/ mouse dose was lethal for their mice, attributing that fact to vein embolism, so they used the $5 \times 10^{5}$ cells/mouse dose, while Park et al. recommended the use of an even higher dose $-2 \times 10^{6} \mathrm{MSCs} / \mathrm{mouse}^{24,25}$ We used triple maximum dose of MSCs/mouse to inject in a single hamster, according to their weight.

After transplantation, the livers of the MSCs-injected groups of both animal models showed engraftment with human hepatocyte-like cells, as proven by the cytoplasmic expression of human AFP, Hep Par 1, CK18, CK7, and OV6). Liver engraftment with human hepatocyte-like cells denoted the homing of MSCs to injured livers and the ability of MSCs to differentiate into hepatocytes. ${ }^{24,25}$

Salem and Thiemermann stated that the therapeutic potentialities of stem cells were based on their tendency to home to the sites of inflammation following tissue injury when introduced IV. ${ }^{26}$ While the mechanisms driving this property were not fully understood, Ley et al. found that the injured cells were induced to express specific receptors or ligands that could facilitate trafficking, adhesion and infiltration of MSCs to the damaged sites, in a way very similar to leukocytes. ${ }^{27}$ Furthermore, it has been demonstrated that MSCs express receptors for chemokines and ligands involved in leukocyte migration during inflammation, including SDF-1 and CXCR4. ${ }^{28,29}$ Hepatic growth factor (HGF) secreted by liver-engrafted MSCs could promote their trans-differentiation into parenchymal hepatocytes. ${ }^{30}$

Our results showed a significant reduction in fibrotic index and improvement in liver function tests following MSC transplantation in both animal models in comparison to the corresponding pathological controls, which was more evident 12 weeks after injection. Many experimental reports showed a promising outcome of MSCs transplantation, as they have the potential to almost completely restore liver function, ameliorate symptoms and enhance survival rates in many hepatic disorders. ${ }^{24,31}$

Mesenchymal stem cells can prevent the hepatocytes from undergoing fibrogenesis by secreting a variety of cytokines, such as HGF, IL- 6 and IL-10. ${ }^{32}$ It has been proven that HGF has anti-apoptotic activity in hepatocytes and plays an essential role in liver regeneration. ${ }^{33,34} \mathrm{Ad}-$ ditionally, MSCs were found to have the potential to attenuate fibrosis in a $\mathrm{CCl}_{4}$-induced liver fibrogenesis animal model by directly suppressing hepatic stellate cells. ${ }^{35}$

In the current study, the liver sections obtained from S. mansoni-infected mice showed large fibrocellular granuloma. Transplantation with $1 \times 10^{6} \mathrm{MSCs}$ after S. mansoni infection showed a diminished granuloma size and a relative decrease in hepatic fibrosis, starting from the $4^{\text {th }}$ week post-transplantation, and reaching its maximum improvement level 12 weeks after MSCs injection. Similar to our findings, Abdel Aziz et al. reported a significant reduction in the hepatic collagen content of hepatic fibrosis 4 weeks post-administration of MSCs and attributed this finding to a modulation in the expression of the MMP and TIMP encoding genes. ${ }^{36}$ Several animal studies and clinical trials stated that MSCs have the potential to reverse the fibrogenesis process by inhibiting collagen deposition and TGF- $\beta 1$ production. ${ }^{9}$ Four weeks post-MSCs transplantation in the Schistosoma model, we detected the engraftment of MSCs into the fibrotic livers with evidence of some signs of regeneration, such as the appearance of newly formed hepatocytes, while the most prominent improvement in mice liver functions came 12 weeks after MSC injection. We found that the IV route was more effective in reducing the fibrosis index and improving liver function than the $\mathrm{IH}$ route, as did Kuo et al., who demonstrated that IV injection was more effective in rescuing liver failure than intrasplenic transplantation. ${ }^{37}$

It was observed that the improvement in both fibrosis index and liver function was more obvious in the $\mathrm{CCl}_{4}$. model than in the S. mansoni-infected model. This could be attributed to the underlying pathology in each model, as $\mathrm{CCl}_{4}$, a metabolite produced by cytochrome P-450 in hepatocytes, leads to lipid peroxidation and membrane damage, which results in a reversible acute centrilobular liver necrosis. ${ }^{38}$ Meanwhile, in the S. mansoni model, most of the pathology is attributed to the host's reaction to the eggs, which reaches its peak by the $8^{\text {th }}$ week of infection, and to the mice not receiving any treatment, so cumulative damage could have occurred. This balance is influenced by the varying competence of the host to kill worms, to inhibit worm fecundity and to destroy eggs and repair tissue damage. ${ }^{39,40}$

In conclusion, CB-MSCs transplantation succeeded in ameliorating liver fibrosis in both Schistosoma and $\mathrm{CCl}_{4}$ chronic liver injury experimental models, as evidenced by the engraftment of the fibrotic livers with newly formed hepatocytes, diminished hepatic fibrosis and fibrotic index of the liver sections, and improvement in liver functions. Our results provide hope that CB-MSCs could be introduced as multipotent stem cells with great potentiality in cell-based therapy of liver fibrosis.

\section{References}

1. Pellicoro A, Ramachandran P, Iredale JP, Fallowfield JA. Liver fibrosis and repair: Immune regulation of wound healing in a solid organ. Nat Rev Immunol. 2014;14(3):181-194.

2. Zhao W, Li J, Cao D, et al. Intravenous injection of mesenchymal stem cells is effective in treating liver fibrosis. World J Gastroenterol. 2012;18(10):1048-1058.

3. Hua J, Qiu P, Zhu H. Multipotent mesenchymal stem cells (MSCs) from human umbilical cord: Potential differentiation of germ cells. Afr J Biochem Res. 2011;5:113-123.

4. Prasajak P, Leeanansaksiri W. Mesenchymal stem cells: Current clinical applications and therapeutic potential in liver diseases. J Bone Marrow Res. 2014;2:137.

5. Kögler G, Sensken S, Airey JA, et al. A new human somatic stem cell from placental cord blood with intrinsic pluripotent differentiation potential. J Exp Med. 2004;200(2):123-135.

6. Kögler G, Radke TF, Lefort A, et al. Cytokine production and hematopoiesis supporting activity of cord blood-derived unrestricted somatic stem cells. Exp Hematol. 2005;33(5):573-583. 
7. Acosta S, Franzese N, Staples M, et al. Human umbilical cord blood for transplantation therapy in myocardial infarction. J Stem Cell Res Ther. 2013;4(Suppl 4):S4-005.

8. Banas A, Teratani T, Yamamoto Y, et al. IFATS collection: In vivo therapeutic potential of human adipose tissue mesenchymal stem cells after transplantation into mice with liver injury. Stem Cells. 2008;26(10):2705-2712.

9. Tsai PC, Fu TW, Chen YM, et al. The therapeutic potential of human umbilical mesenchymal stem cells from Wharton's jelly in the treatment of rat liver fibrosis. Liver Transpl. 2009;15(5):484-495.

10. Broxmeyer HE, Douglas GW, Hangoc G, et al. Human umbilical cord blood as a potential source of transplantable hematopoietic stem/ progenitor cells. Proc Natl Acad Sci USA. 1989;86(10):3828-3832.

11. Kögler G, Callejas J, Hakenberg P, et al. Hematopoietic transplant potential of unrelated cord blood: Critical issues. $J$ Hematother. 1996;5(2):105-116.

12. Zaibak F, Bello P, Kozlovski J, et al. Unrestricted somatic stem cells from human umbilical cord blood grow in serum-free medium as spheres. BMC Biotechnol. 2009;9:101.

13. Demerdash Z, El Baz H, Mahmoud F, et al. Enhancing ex vivo expansion of cord blood-derived unrestricted somatic stem cells for clinical applications. Cell Prolif. 2013;46(6):628-636.

14. Waclawczyk S, Buchheiser A, Flögel U, Radke TF, Kögler G. In vitro differentiation of unrestricted somatic stem cells into functional hepatic-like cells displaying a hepatocyte like glucose metabolism. J Cell Physiol. 2010;225(2):545-554.

15. Moharib MN, Hammam OA, Salman FH, El-naggar MM, Sherif SA. Transplantation of modified and fresh hepatocyte reduces hepatotoxicity induced by carbon tetrachloride. Life Sci J. 2014;11:641-649.

16. German G, Hytiroglou P, Fotiadu A, Burroughs A, Dhillon A. Assessment of fibrosis and cirrhosis in liver biopsies. Semin Liver Dis. 2011;31(1):82-90.

17. Tsai CC, Hung SC. Functional roles of pluripotency transcription factors in mesenchymal stem cells. Cell Cycle. 2012;11(20):3711-3712.

18. Foroutan T, Sadeghizadeh M. Lower oncogenic potential of human mesenchymal stem cells derived from cord blood compared to bone marrow and adipose tissues. Indian J Appl Res. 2014;4(9):23-27.

19. Liang XJ, Chen XJ, Yang DH, Huang SM, Sun GD, Chen YP. Differentiation of human umbilical cord mesenchymal stem cells into hepatocyte-like cells by hTERT gene transfection in vitro. Cell Biol Int 2012;36(2):215-221.

20. Li D, Fan J, He X, et al. Therapeutic effect comparison of hepatocytelike cells and bone marrow mesenchymal stem cells in acute liver failure of rats. Int J Clin Exp Pathol. 2015;8(1):11-24

21. Hong J, Jin $\mathrm{H}$, Han J, et al. Infusion of human umbilical cord-derived mesenchymal stem cells effectively relieves liver cirrhosis in DEN-induced rats. Mol Med Rep. 2014;9(4):1103-1111.

22. Li J, Zhang L, Xin J, et al. Immediate intraportal transplantation of human bone marrow mesenchymal stem cells prevents death from fulminant hepatic failure in pigs. Hepatology. 2012;56(3):1044-1052.

23. Shams S, Mohsin S, Nasir GA, Khan M, Khan SN. Mesenchymal stem cells pretreated with HGF and FGF4 can reduce liver fibrosis in mice. Stem Cells Int. 2015;2015:747245.
24. Yang JF, Cao HC, Pan QL, Yu J, Li J, Li LJ. Mesenchymal stem cells from the human umbilical cord ameliorate fulminant hepatic failure and increase survival in mice. Hepatobiliary Pancreat Dis Int. 2015;14(2):186-193.

25. Park M, Kim YH, Woo SY, et al. Tonsil-derived mesenchymal stem cells ameliorate $\mathrm{CCl} 4$-induced liver fibrosis in mice via autophagy activation. Sci Rep. 2015;5:8616.

26. Salem HK and Thiemermann C. Mesenchymal stromal cells: Current understanding and clinical status. Stem Cells. 2010;28(3):585-596.

27. Ley K, Laudanna C, Cybulsky MI, Nourshargh S. Getting to the site of inflammation: The leukocyte adhesion cascade updated. Nat Rev Immunol 2007;7(9):678-689.

28. Honczarenko M, Le Y, Swierkowski M, Ghiran I, Glodek AM, Silberstein LE. Human bone marrow stromal cells express a distinct set of biologically functional chemokine receptors. Stem Cells. 2006;24(4):1030-1041.

29. Ringe J, Strassburg S, Neumann K, et al. Towards in situ tissue repair: Human mesenchymal stem cells express chemokine receptors CXCR1, CXCR2 and CCR2, and migrate upon stimulation with CXCL8 but not CCL2. J Cell Biochem. 2007;101(1):135-146.

30. Liu WH, Song FQ, Ren LN, et al. The multiple functional roles of mesenchymal stem cells in participating in treating liver diseases. J Cell Mol Med. 2015;19(3):511-520.

31. Banas A, Teratani T, Yamamoto Y, et al. Rapid hepatic fate specification of adipose-derived stem cells and their therapeutic potential for liver failure. J Gastroenterol Hepatol. 2009;24(1):70-77.

32. Prosser CC, Yen RD, Wu J. Molecular therapy for hepatic injury and fibrosis: Where are we? World J Gastroenterol. 2006;12(4):509-515.

33. George J, Rao KR, Stern R, Chandrakasan G. Dimethylnitrosamineinduced liver injury in rats: The early deposition of collagen. Toxicology. 2001;156(2-3):129-138.

34. Matsuda-Hashii $\mathrm{Y}$, Takai K, Ohta $\mathrm{H}$, et al. Hepatocyte growth factor plays roles in the induction and autocrine maintenance of bone marrow stromal cell IL-11, SDF-1 alpha, and stem cell factor. Exp Hematol. 2004;32(10):955-961.

35. Pan RL, Wang P, Xiang LX, Shao JZ. Delta-like1 serves as a new target and contributor to liver fibrosis down-regulated by mesenchymal stem cell transplantation. J Biol Chem. 2011;286(14):12340-12348.

36. Abdel Aziz MT, Atta HM, Roshdy NK, et al. Amelioration of murine Schistosoma mansoni induced liver fibrosis by mesenchymal stem cells. J Stem Cells Regen Med. 2012;8(1):28-34.

37. Kuo TK, Hung SP, Chuang $\mathrm{CH}$, et al. Stem cell therapy for liver disease: Parameters governing the success of using bone marrow mesenchymal stem cells. Gastroenterology. 2008;134(7):2111-2121.

38. Hayashi H, Sakai T. Animal models for the study of liver fibrosis: New insights from knockout mouse models. Am J Physiol Gastrointest Liver Physiol. 2011;300(5):G729-738.

39. Chensue SW, Boros DL. Modulation of granulomatous hypersensitivity. I. Characterization of T lymphocytes involved in the adoptive suppression of granuloma formation in Schistosoma mansoni-infected mice. J Immunol. 1979;123(3):1409-1414.

40. Cheever AW, Lenzi JA, Lenzi HL, Andrade ZA. Experimental models of Schistosoma mansoni infection. Mem Inst Oswaldo Cruz. 2002;97(7):917-940. 